\title{
COMITÊS DE ÉTICA EM PESQUISA: ADEQUAÇÃO À RESOLUÇÃO 196/96
}

\author{
Ellen Hardy*, Silvana Ferreira Bento, Maria José Duarte Osis, Eliana Maria Hebling \\ Trabalho realizado no Departamento de Tocoginecologia da Faculdade de Ciências Médicas da Universidade \\ Estadual de Campinas - UNICAMP e Centro de Pesquisas Materno-Infantis de Campinas - Cemicamp, Campinas, SP
}

RESUMO - OBJETIVO. Este artigo apresenta a avaliação da estrutura, funcionamento e atuação de 17 Comitês de Ética em Pesquisa, na opinião de seus presidentes, considerando as determinações da Resolução 196/96 do Conselho Nacional de Saúde, Ministério da Saúde, Brasil.

Métodos. Foram identificados os presidentes de 33 Comitês que avaliavam projetos de pesquisa em regulação da fecundidade. Eles foram indicados pelos responsáveis dos serviços de ginecologia de 46 faculdades de medicina no Brasil e pelos diretores de quatro centros de pesquisa em reprodução humana. Uma carta foi enviada aos presidentes, convidando-os a participar voluntariamente de uma pesquisa, preenchendo um questionário.

RESULTADOs. Dezessete presidentes responderam o questionário. Os resultados mostraram uma série de violações à Resolução 196/96. Três Comitês não tinham representantes da comunidade; quatro demoravam mais de um mês para emitir o parecer final dos protocolos e 13 não acompanhavam o desenvolvimento dos projetos. A composição e arquivamento dos protocolos estavam de acordo com a Resolução, porém, o tempo de mandato era diferente do estabelecido em oito dos Comitês avaliados. Quase todos os presidentes (entre 14 e 17) consideraram a composição e atuação de seus CEPs adequados. A grande maioria dos presidentes (II) qualificou a Resolução como sendo apropriada, porém, difícil de ser cumprida.

ConCLUSÃo. Os resultados sugerem que um amplo debate sobre a viabilidade operacional da Resolução seria oportuno. Este processo resultaria em sugestões valiosas para 0 aperfeiçoamento e aplicabilidade das normas. Isto contribuiria para a melhoria da qualidade cientifica e ética dos estudos desenvolvidos no Brasil.

Unitermos: Resolução 196/96. Comitês de Ética em Pesquisa. Pesquisa.

\section{INTRODUÇÃO}

Os princípios éticos que orientam pesquisas que envolvem seres humanos têm sido alvo de constantes discussões, principalmente quanto a possíveis abusos por parte da comunidade científical,2,3. Os debates envolvendo a temática da ética médica não são recentes e parecem ter encontrado expressão já no século XIX, quando, em 1803, o médico inglês Thomas Percival propôs o primeiro código ético estabelecendo preceitos morais para a prática da medicina e da experimentação clínica4. Em seu livro "Medical Ethics", o Dr. Percival propôs que, quando um médico desejasse experimentar um novo medicamento, deveria consultar previamente outros colegas ${ }^{5}$. Suas propostas deram origem à criação de órgãos colegiados para discussão de novos procedimentos que seriam realizados em pacientes de hospitais.

Em 1947, o Código de Nuremberg, emitido na Alemanha pelos juízes do tribunal que

\footnotetext{
*Correspondência:

Caixa Postal 6181

CEP: 13084-971 - Campinas - SP

E-mail: hardy@unicamp.br
}

julgou médicos nazistas contra abusos cometidos em pesquisas experimentais envolvendo seres humanos, incluiu, pela primeira vez, algumas diretrizes éticas a esse respeito. Este documento propôs como essencial o consentimento voluntário do sujeito de pesquisa $a^{5}$. Nos anos subseqüentes, o conteúdo ético deste Código passou por uma ampliação, resultando na Declaração de Helsinque, adotada pela Associação Médica Mundial em 19647. Este documento, em sua revisão de 1975, fez referência à criação de comitês independentes para análise ética de projetos de pesquisa com seres humanos ${ }^{7,8}$. A última versão da Declaração de Helsinque, em 2000, apresenta como exigência para qualquer pesquisa que o investigador submeta seu protocolo à "aprovação de um comitê de avaliação ética especialmente designado, que deve ser independente do pesquisador, do patrocinador ou de qualquer outro tipo de influência indevida"'.

No Brasil, as comissões de ética parecem ter surgido em 1985, quando o Conselho Federal de Medicina (CFM) publicou a Resolução $1215 / 85$. Este documento determinava que os Conselhos Regionais de Medicina (CRMs) criassem Comissões de Ética Médica
(CEMs) em todos os estabelecimentos ou entidades, sob sua jurisdição, onde a medicina era exercida ${ }^{10}$. Em 1986, o CRM de São Paulo, através da Resolução 023/86, padronizou os critérios de criação, competência e procedimentos dessas Comissões"'. Segundo esse documento, uma das atribuições das CEMs era "opinar sobre todos os projetos de investigação médica realizados na instituiçã̃o e que envolvam seres humanos".

Em 1988, o Conselho Nacional de Saúde (CNS), do Ministério da Saúde, publicou a Resolução 01/88. Uma das determinações desta Resolução era que toda instituição de saúde que realizava pesquisas em seres humanos, credenciada pelo CNS, tivesse um Comitê de Ética. Algumas das principais atribuições desse Comitê eram autorizar a realização de pesquisas em seres humanos e orientar os pesquisadores quanto aos aspectos éticos e de segurança biológica. O CNS determinava: "a pesquisa somente poderá ser iniciada após parecer favorável, por escrito, do Comitê de Ética e do Comitê de Segurança Biológica, conforme o caso, tendo informado ao responsável pela instituição de atenção à saúde" 12 . Entretanto, nos anos seguintes, houve diversas 
denúncias de abusos, cometidos por alguns pesquisadores, à integridade da mulher em pesquisas sobre contraceptivos ${ }^{13-16}$.

Em 1996, após exaustivo trabalho de revisão da Resolução 0।/88, o CNS publicou a Resolução 196/96 ${ }^{17}$, determinando que toda e qualquer pesquisa com seres humanos, não somente as da área biomédica, devem ser aprovadas por um Comitê de Ética em Pesquisa (CEP). Essa Resolução, no Capítulo VII, estabelece as normas a serem seguidas pelos CEP na sua organização, atribuiç̧ões e atuação.

Considerando que no Brasil os CEPs são regidos pelo Comitê Nacional de Ética em Pesquisa (CONEP), Ministério da Saúde, espera-se que eles sigam as normas preconizadas pela Resolução 196/96. Daí o interesse em verificar em que medida os CEPs, criados nos últimos anos, conseguem cumprir com suas disposições. Como a maior parte das denúncias de violações éticas referiam-se a pesquisas na área da regulação da fecundidade ${ }^{13-16}, 0$ presente estudo limitou-se a avaliar CEPs que atuavam nessa mesma área.

\section{Métodos}

Foram identificados os presidentes de 33 CEPs que avaliavam projetos de pesquisa em regulação da fecundidade. A identificação desses presidentes foi obtida a partir de: I) informação proporcionada pelos 46 responsáveis pela área de ginecologia das universidades que possuíam o curso de medicina, de acordo com levantamento do Conselho Federal de Medicina $\left(\right.$ CFM) ${ }^{18} ; 2$ ) pelos diretores dos quatro centros de pesquisa em regulação da fecundidade, segundo o Inventario Latinoamericano de Centros de Investigaciones, Enseñanza y Recursos Humanos en Reproducción Humana $(\mathrm{PLACIRH})^{19}$. Foi enviada uma carta a esses 33 presidentes, convidando-os a participar voluntariamente de uma pesquisa. A carta esclarecia - objetivo, assegurava o sigilo e solicitava 0 preenchimento de um questionário, enviado anexo. Este continha perguntas que seguiam, principalmente, as disposições da Resolução 196/96, sobre a estrutura e o funcionamento do seu CEP e sobre como era feita a revisão dos protocolos. Também foi solicitada sua opinião acerca da atuação do CEP que presidia. O questionário foi pré-testado no CEP de uma universidade e esses resultados não foram incluídos na análise. Foi priorizada a utilização do correio eletrônico, tanto para o envio como para o recebimento das correspondências. Ocasionalmente foi necessário utilizar os serviços da Empresa de Correios e Telégrafos e o fax. Nos casos de não obtenção de resposta num período de 15 dias, foram feitas, pelo menos, três outras tentativas de contato por correio eletrônico, telefone, fax e/ou telegrama. Dos 33 presidentes, mais da metade (52\%) respondeu o questionário, três se recusaram a responder e 13 não deram nenhum retorno.

Cada questionário preenchido foi revisado para verificar se todas as perguntas estavam respondidas e devidamente registradas nos espaços a elas destinados. Quando necessário, foi feito novo contato para esclarecimento de dúvidas e/ou obtenção de respostas que faltavam. Para assegurar o sigilo dos participantes, os questionários foram identificados apenas por números e os dados que permitiriam saber sua procedência (endereço de correio eletrônico ou remetente) foram removidos e destruídos assim que os questionários foram revisados. Finalmente, após o banco de dados estar completo, a numeração dos questionários foi refeito, alocando-se um número aleatório a cada presidente, o que tornou inviável sua posterior identificação.

Para digitar as respostas a partir do próprio questionário, foi preparado um banco de dados no SPSS. Os dados foram digitados duas vezes, por pessoas diferentes, permitindo a verificação simultânea da digitação. Além disso, foi feita uma verificação manual das freqüências das variáveis para identificar e corrigir possíveis erros de consistência. A análise dos dados foi descritiva. Somente as informações apresentadas na Tabela 6 não correspondem a disposiç̧ões da Resolução 196/96. Elas foram coletadas com base na experiência prática de uma das pesquisadoras, que havia sido membro de dois CEPs e também presidente de um deles.

Dado o objetivo do estudo, não houve um Termo de Consentimento Livre e Esclarecido a ser assinado, porque isto poderia sugerir as respostas para muitas das perguntas do questionário enviado. As pessoas foram convidadas a participar voluntariamente e esclarecidas a respeito da pesquisa através de carta-convite, na qual se assegurou a manutenção do sigilo. $O$ fato de responderem ao questionário foi entendido como o consentimento em participar da pesquisa. Essa medida foi incluída no protocolo da pesquisa, que foi aprovado pela Comissão de Pesquisa do Departamento de Tocoginecologia do Centro de Assistência Integral à Saúde da Mulher (CAISM) e pelo CEP da Faculdade de Ciências Médicas da Universidade Estadual de Campinas - UNICAMP.

\section{Resultados}

Perfil dos presidentes - Treze dos 17 presidentes eram do sexo masculino e dez eram médicos. Sete tinham doutorado e quatro pósdoutorado. A maioria (13) presidia o CEP há mais de 12 meses (Tabela I). Todos os presidentes referiram conhecer a Resolução 196/ 96. A grande maioria ( I 6) disse tê-la lido inteira, com atenção. Doze deles consideraram-na adequada, porém, difícil de ser cumprida. Segundo as informações dos presidentes, a maior parte dos CEPs ( I 5) pertencia a universidades, das quais oito eram federais. Nove CEPs avaliavam somente projetos da própria instituição e os outros analisavam também protocolos de outras instituições.

\section{Cumprimento da Resolução 196/96 -}

Em relação à composição dos CEPs, ainda que em todos eles houvesse representantes de diferentes áreas do conhecimento, em três não havia membros da comunidade, conforme exige a Resolução (Cap. VII, item 4). Nenhum deles possuía menos de sete membros, mas, em nove dos 17, nunca havia sido solicitada a colaboração de um consultor add hoc (Cap.VII, item 5). Todos os CEPs eram compostos por pessoas de ambos os sexos, de acordo com a Resolução, mas em quase a metade deles $(8 / 17)$ não se cumpria 0 tempo de mandato dos membros de três anos, de acordo com a Resolução (Cap. VII, item 9). Em todos os CEPs a reeleiçãa dos membros era permitida. Sobre a escolha dos membros, todos os I 5 presidentes que responderam este item informaram que seus CEPs cumpriam com a exigência de que, pelo menos, 50\% de seus membros tivessem experiência em pesquisa. (Tabela 2).

Quanto aos documentos exigidos para avaliação de protocolo, quatro presidentes referiram que seus CEPs não solicitavam o currículo dos pesquisadores principais e oito não solicitavam o currículo dos demais pesquisadores. Todos os presidentes afirmaram que 
Tabela I - Perfil dos presidentes (números absolutos)

\begin{tabular}{|c|c|}
\hline Sexo & \\
\hline $\begin{array}{l}\text { Masculino } \\
\text { Feminino }\end{array}$ & $\begin{array}{l}13 \\
4\end{array}$ \\
\hline $\begin{array}{l}\text { Formação profissional } \\
\text { Médico } \\
\text { Psicólogo } \\
\text { Enfermeiro } \\
\text { Dentista } \\
\text { Cirurgião dentista } \\
\text { Biomédico } \\
\text { Farmacêutico }\end{array}$ & $\begin{array}{l}10 \\
2 \\
1 \\
1 \\
1 \\
1 \\
1\end{array}$ \\
\hline $\begin{array}{l}\text { Escolaridade } \\
\text { Superior } \\
\text { Mestrado } \\
\text { Doutorado } \\
\text { Pós-doutorado }\end{array}$ & $\begin{array}{l}3 \\
3 \\
7 \\
4\end{array}$ \\
\hline $\begin{array}{l}\text { Tempoque presidia o CEP } \\
\text { Até } 6 \text { meses } \\
7-12 \text { meses } \\
13 \text { ou mais }\end{array}$ & $\begin{array}{l}3 \\
1 \\
13\end{array}$ \\
\hline Total de presidentes & 17 \\
\hline
\end{tabular}

Tabela 2-Composição dos CEPS, mandato e escolha dos membros (números absolutos)

\section{Composição}

Possuía membros de diferentes áreas do

conhecimento

Possuía membros representantes da comunidade

Possuía sete ou mais membros

Solicitada colaboração de consultor "ad hoc"

Possuíamembros de ambosos sexos

17

14

17

8

17

\section{Mandato e escolha dos membros}

Duração de três anos\#

Permitida a recondução/reeleição

Metade ou mais tinha experiência em pesquisa*

Total de presidentes

Faltou a informação de: *dois presidentes; \# um presidente

Tabela 3 - Documentos que eram exigidos sempre para a avaliação de protocolos (números absolutos)

$$
\begin{aligned}
& \text { Currículo do pesquisador principal } \\
& \text { Currículos dos demais pesquisadores } \\
& \text { Folha de rosto (exigida pela CONEP) } \\
& \text { Projeto escrito em português } \\
& \text { Cópia do TCLE } \\
& \text { Orçamento financeiro detalhado }
\end{aligned}
$$

Total de presidentes a folha de rosto exigida pela CONEP era sempre solicitada; 16 disseram que sempre requeriam o projeto de pesquisa escrito em português e uma cópia do Termo de Consentimento Livre e Esclarecido (TCLE). Apenas nove CEPs solicitavam sempre o orçamento financeiro detalhado (Tabela 3).

Os antecedentes científicos, mais especificamente a revisão da literatura, não eram avaliados em oito dos 17 CEPs. Cinco CEPs não revisavam as hipóteses e nem a remuneração do pesquisador. Todos os presidentes referiram que o avaliador sempre revisava os aspectos éticos, mas, em um dos CEP, o TCLE não era revisado. Em 16 CEPs, a justificativa e os objetivos eram sempre revisados e, em 15 , os itens relativos a sujeitos de pesquisa e métodos também eram (Tabela 4).

Sobre as atribuiçõ̃es dos CEPs, dispostas na Resolução 196/96 (Cap.VII, item 13), I 4 presidentes disseram que obedeciam à determinação de que o parecer sobre cada protocolo era sempre consubstanciado, por escrito. Entretanto, 13 dos 17 presidentes declararam que seus CEPs nem sempre requeriam relatórios anuais das pesquisas para acompanhamento. Quatro presidentes referiram que o tempo de demora para enviar um parecer era maior do que um mês, tempo previsto pela Resolução 196/96, Cap. VII, item 13b. Dezesseis presidentes declararam sempre manter a guarda confidencial de todos os dados obtidos na execução de sua tarefa. Quinze referiram que sempre arquivavam os protocolos por cinco anos e que mantinham comunicação regular e permanente com a CONEP. A grande maioria dos presidentes disse que nunca houve necessidade de requerer sindicâncias, nem de comunicar irregularidades éticas à CONEP; também não haviam recebido denúncias de abusos, nem de fatos adversos (Tabela 5).

Outras características avaliadas - Oito presidentes relataram que, em seus CEPs, o parecer para cada protocolo era emitido apenas por um membro. Dos nove que declararam que o parecer era emitido por mais de um membro, estes possuíam formações acadêmicas distintas. Apenas três presidentes disseram que o parecerista nunca podia entrar em contato com o pesquisador principal, e seis referiram que nunca os pesquisadores sabiam quem havia emitido o parecer sobre o seu protocolo. Dos 17 presidentes, três relataram que 


\begin{tabular}{lc}
\hline Tabela 4 - Itens que sempre eram revisados pelos pareceristas (números absolutos) \\
\hline Antecedentescientfícos & 9 \\
Hipóteses & 12 \\
Remuneração do pesquisador & 12 \\
Aspectoséticos & 17 \\
TCLE & 16 \\
Objetivos & 16 \\
Justificativa & 16 \\
Sujeitos & 15 \\
Métodos & 15 \\
Total de presidentes & 17 \\
\hline
\end{tabular}

Tabela 5 - Frequiência com que os 17 CEPs cumpriam diferentes atribuições (números absolutos)

\begin{tabular}{|c|c|c|c|c|}
\hline Atribuições & Sempre & Àsvezes & Nunca & $\begin{array}{l}\text { Nunca foi } \\
\text { necessário }\end{array}$ \\
\hline \multicolumn{5}{|l|}{ a) Sempre aplicáveis } \\
\hline O parecer era consubstanciado & 14 & 3 & 0 & 0 \\
\hline $\begin{array}{l}\text { O tempo médio para enviar o } \\
\text { parecer final era de até um mês }\end{array}$ & 13 & 0 & 4 & 0 \\
\hline $\begin{array}{l}\text { Manter a guarda confidencial dos } \\
\text { dados obtidos na execuçąa de sua tarefa }\end{array}$ & 16 & I & 0 & 0 \\
\hline $\begin{array}{l}\text { Arquivar os protocolos por cinco anos } \\
\text { após o encerramento dos estudos" }\end{array}$ & 15 & I & 0 & 0 \\
\hline $\begin{array}{l}\text { Manter comunicação regulare } \\
\text { permanente coma CONEP }\end{array}$ & 15 & I & 0 & I \\
\hline $\begin{array}{l}\text { Acompanhar os projetos através de } \\
\text { relatórios anuais dos pesquisadores }\end{array}$ & 4 & 10 & 2 & I \\
\hline \multicolumn{5}{|l|}{ b) Aplicáveis, se necessário } \\
\hline $\begin{array}{l}\text { Decidir pela continuidade, modificação } \\
\text { ou suspensão de uma pesquisa }\end{array}$ & 5 & 4 & 2 & 6 \\
\hline $\begin{array}{l}\text { Requerer sindicância frente a denúncias } \\
\text { de irregularidades éticas em uma pesquisa }\end{array}$ & 2 & 0 & 3 & 12 \\
\hline $\begin{array}{l}\text { Em caso de comprovação de } \\
\text { irregularidades, comunicar ofato à CONEP* }\end{array}$ & 2 & 0 & 2 & 12 \\
\hline $\begin{array}{l}\text { Receber denúncias de abusos e/ou } \\
\text { de fatos adversos, por parte dos sujeitos } \\
\text { de pesquisa* }\end{array}$ & I & I & 3 & 11 \\
\hline
\end{tabular}

Faltou a informação de: * um presidente

nem sempre os projetos avaliados eram apresentados e discutidos na reunião do CEP (Tabela 6).

Opinião dos presidentes sobre o funcionamento dos CEPs - A maior parte dos presidentes ( 14 a |7) considerou adequada a atuação de seus CEPs nos seguintes aspectos: número total e áreas do conhecimento representadas pelos seus membros; duração e forma de renovação dos mandatos; número de membros que avaliavam cada protocolo; itens que eram revisados nos protocolos e tempo que levavam para emitir um parecer (Tabela 7).

Segundo a Resolução 196/96, a revisão de cada protocolo deve culminar no seu enquadramento em uma dessas categorias: aprovado; aprovado com pendência; retirado; não aprovado; aprovado e encaminhado, com o devido parecer, para a CONEP. Foi solicitado aos presidentes que escrevessem sua opinião sobre este enquadramento. Onze presidentes consideraram-no adequado; um considerou aceitável; três apresentaram sugestões relacionadas ao item "aprovado com pendência", principalmente descrevendo a atuação de seu CEP frente a este enquadramento, e dois fizeram comentários gerais sobre como seus CEPs atuam. Algumas das respostas foram as seguintes:
"Considero adequada e, mais ainda, após a complementação das pendências, deverse-ia encaminhar carta com parecer final (há pesquisadores que iniciam a pesquisa ainda que o protocolo esteja com pendências...) (Nós fazemos carta de tal forma que o fato não ocorra)".

"Adequado. Em relação ao item e: 'Aprovado e encaminhado... para a CONEP', a necessidade de apreciação pela CONEP deve se tornar cada dia mais excepcional, à medida que os CEPs se tornem mais competentes em sua avaliação".

"É o ideal, pois impede que muitos projetos sejam reprovados na primeira avaliação, também possui um caráter educacional, pois muitas vezes o pesquisador não tinha refletido sobre uma necessidade ou aspecto ético, assim, quando o CEP aponta um problema ético, ele possui a oportunidade de modificar sua visão frente ao mesmo e ainda pode executar sua pesquisa".

\section{Discussão}

Os resultados colocam em evidência as dificuldades que alguns CEPs ainda tinham para cumprir as disposições da Resolução 196/96. Aparentemente, resultava mais fácil cumprir as disposições com relação ao número e sexo dos membros, áreas do conhecimento representadas por eles, permitir a reeleição, bem como ter mais da metade dos membros com experiência em pesquisa.

A demora de alguns CEPs na emissão de um parecer, além do período estabelecido pela Resolução, pode ter várias explicações. $\bigcirc$ CEP ter uma demanda superior à sua capacidade de avaliação, os membros terem outras prioridades ou falta de experiência em realizar esse trabalho. A demora maior representa um problema que necessita solução, pois causa constrangimento ao CEP, que se vê obrigado a dar satisfações. Ao mesmo tempo, a demora causa dificuldades aos pesquisadores, que ficam impossibilitados de começar o estudo na data prevista. Se os recursos já foram aprovados, terão que informar à agência financiadora de que o início do estudo será adiado. Além disso, se os recursos concedidos estão expressos em reais, corre-se o risco de sua desvalorização, inviabilizando a realização da pesquisa. Também é possível que no estudo se vá utilizar algum produto cuja validade possa vencer antes de terminar o trabalho de campo. 
em evidência que pelo menos alguns CEPs não conseguem aplicar, na prática, todas as disposições da Resolução 196/96 e, ainda, os

Tabela 6 - Características da avaliação de protocolos (números absolutos)

Número de membros que emitiamparecer

Só um 8

Mais de um

8
$9 *$

*Formação acadêmica distinta em cinco dos nove

O parecerista podia contatar o pesquisador principal

Sempre

Àsvezes

Nunca

4

Os pesquisadores sabiam quem emitiu o parecer

Sempre

Ásvezes

Nunca

Parecer final

Sempre apresentado e discutido na reunião do CEP

Alguns são apresentados e discutidos e outros apenas homologados

Total de presidentes
17

\begin{tabular}{cc}
\hline Tabela 7 - Procedimentos que os presidentes consideravam adequados em seus CEPs (números absolutos) \\
\hline Número total de membros & 16 \\
Áreas do conhecimento representadas pelos membros & 16 \\
Duração do mandato dos membros & 15 \\
Forma de renovação dos membros & 14 \\
Número de membros que avaliavam cada protocolo & 17 \\
Itens que eram revisados & 16 \\
Tempo para emitír um parecer & 15 \\
Total de presidentes & 17 \\
\hline
\end{tabular}

A possível sobrecarga dos CEPs também pode explicar porque a revisão sistemática de todos os itens prescritos pela Resolução nem sempre era feita. Em especial, evidenciou-se a falha em revisar os antecedentes científicos. Cabe ressaltar que, segundo a Resolução, "a revisão ética de toda e qualquer proposta de pesquisa envolvendo seres humanos não poderá ser dissociada da sua análise científica" (Cap. VII, item I 4a). Quando se revisa somente os aspectos éticos e não os metodológicos existe o risco de que os resultados finais, publicados, não reflitam a realidade dos fatos. Isso pode causar danos às pessoas, caso outros pesquisadores utilizem esses resultados equivocados como base para outros estudos ou tratamento de pacientes.

Outro aspecto que se apresentou diferente do estabelecido pela Resolução 196/96 foi o não acompanhamento do desenvolvimento dos projetos. Isto significa que esses CEPS não sabiam se os estudos estavam sendo realizados de acordo com o protocolo aprovado, nem mesmo se eram descontinuados. A desconti- nuidade, sem justificativa aceita pelo Comitê que aprovou o protocolo, é considerada eticamente inaceitável pela Resolução (Cap. VII, item I 3 f). Entretanto, é preciso ponderar se o fato dos CEPs não estarem realizando a supervisão dos estudos não é outro indicador de sobrecarga de trabalho e/ou de falta de recursos para cumprirem essa atribuição. De qualquer maneira, essa situação requer análise e busca de soluções, na medida que representa uma debilidade operacional que interfere no seguimento dos preceitos da Resolução, cujas conseqüências éticas para os participantes são desconhecidas.

Uma solução já proposta pela Resolução é a utilização de membros add hoc ou consultores add hoc, que ajudariam e aliviariam essa carga, entretanto, mais da metade dos CEPs nunca tinham utilizado este recurso.

É evidente que uma amostra de 17 CEPs, aqui analisada, não é representativa do total dos Comitês funcionando no Brasil. Por isso mesmo, não cabe tirar porcentagens, nem fazer generalizações. Apenas pode-se colocar resultados permitem identificar quais destas disposições parecem ser mais difíceis de ser cumpridas.

Por outro lado, os resultados aqui apresentados foram obtidos através de uma avaliação independente, ou seja, não foi feita pelos próprios CEPs e nem pela CONEP, o que permitiu aos participantes responder com maior liberdade. A porcentagem de presidentes (52\%) que preencheu o questionário foi relativamente elevada, considerando as dificuldades enfrentadas para se obter resposta a pesquisas feitas por correio. Pesquisas realizadas com médicos, por correio, tiveram uma taxa média de resposta de 54\%, em países desenvolvidos, onde existe maior hábito de uso do correio para esta e outras funções ${ }^{20,21}$. Os resultados apresentados neste artigo correspondem às opiniões dos presidentes, e não necessariamente refletem as dos demais membros dos CEPs. Porém, o estudo permitiu que fossem identificadas algumas das dificuldades enfrentadas pelos presidentes no cumprimento das normas da Resolução e que opinassem sobre esta no que diz respeito à atuação dos seus CEPs.

$\vec{E}$ necessário reconhecer o enorme progresso que tem significado para a ética em pesquisa no Brasil a publicação, divulgação e aplicação da Resolução 196/96. A necessidade de criar os CEPs e enviar os protocolos de pesquisa à revisão ética colocam o Brasil vários passos à frente dos demais países da América Latina e de outras regiões menos desenvolvidas. Isto, porém, não significa que o processo não possa se aperfeiçoar ainda mais, que é justamente o objetivo da avaliação aqui apresentada.

Na opinião da maioria dos presidentes, os seus CEPs apresentavam condições adequadas de funcionamento. Isto leva a pensar que, no julgamento desses profissionais, os itens em que, eventualmente, os Comitês não estavam atuando de acordo com a Resolução, não eram considerados tão relevantes para o seu trabalho. Reforça essa perspectiva o fato de que a grande maioria dos presidentes qualificou a Resolução como sendo adequada, porém, difícil de ser cumprida. Em vista dessas considerações, parece apropriado sugerir que, decorridos sete anos da implementação da Resolução, seja oportuno promover um amplo debate entre os profissionais envolvidos no cumprimento dessas normas, para discutirem 
HARDY E ET AL.

sua viabilidade operacional. Isto está de acordo com o Capítulo I da Resolução 196/96, que prevê revisões periódicas da mesma, conforme necessidades identificadas. Um processo como esse resultaria em sugestões valiosas para o aperfeiçoamento e aplicabilidade desse documento e, conseqüentemente, melhoraria a qualidade científica e ética dos estudos desenvolvidos no Brasil.

\section{Agradecimentos}

As autoras agradecem a contribuição financeira da Fundação de Amparo à Pesquisa do Estado de São Paulo (FAPESP) e do Fundo de Apoio ao Ensino e à Pesquisa (FAEP) da Universidade Estadual de Campinas (UNICAMP). Também são gratas a todas as pessoas que, de alguma forma, contribuíram com seu tempo para que esta pesquisa fosse realizada.

Conflito de interesse: não há.

\section{SUMMARY \\ INSTITUTIONAL REVIEW Boards: COMPLIANCE WITH Resolution 196/96}

PURPOSE. This article intends to evaluate the structure, functioning and performance of 17 Institutional Review Boards (IRB), from the viewpoint of their presidents, in relation to the instructions of Resolution 196/96 of the National Council of Health, Ministry of Health, Brazil.

METHODS. Presidents of 33 IRBS, that evaluated proposals for research on fertility control, were indicated by the professionals responsible for the area of gynecology in 46 Brazilian medicalschools and by the directors of four research centers on human reproduction. A letter with a questionnaire were sent to the presidents, asking them to volunteer for the study.

RESULTS. Seventeen presidents completed the questionnaire. Results disclosed a series of violations of Resolution 196/96. Three IRBs did not include representatives of the community among theirmembers; four took overa month to release the Board's decision on proposals and 13 did notfollow-up the studies. Composition and filing of the research proposals were in agreement with the Resolution. However, in eight IRBs time of mandate differed from that set forth in the Resolution. Almost all presidents (about 14 of the 17) thought that the composition and activities of their IRBs were adequate. Eleven considered the Resolution appropriate but difficult to comply with.

CONCLUSION. These results suggest that an extensive debate on the operational viability of the Resolution would be timely. This endeavor could bring forth valuable suggestions for the improvementand applicability of the Resolution contributing to the improvement of the scientific and ethical quality of research. [Rev Assoc Med Bras 2004; 50(4): 457-62]

KEY WORDS: Resolution 196/96. Institutional review boards. Research.

\section{REFERÊNCIAS}

I. Hardy E, Duarte GA, Osis MJD. Aspectos éticos da anticoncepção. In:Andrade RP, Poli M, Petracco A, Melo KM, Camargo A, coordenadores. Contracepção: promoção da saúde sexual e reprodutiva. Rio de Janeiro: Revinter; 2000. p.229-35.

2. Arruda RO. Cientistas discutem ética nas experiências. O Estado de São Paulo, São Paulo. 1996 jul II. Seção: Reunião.

3. Sabbatini RME. "Cobaias humanas" e a experimentação médica. Jornal Correio Popular, Campinas. 1999 jul 2. Available from: http:// home. nib.unicamp.br/ sabbatin

4. Ortiz JS. Ética: clima moral de la práctica médica. Med Clin (Barc) 1999; I 12:696-8. Available from: http://www.atheneum. doyma.es/sala_I/lec03eti.htm.

5. Comitês de Bioética, de 18/04/2002. Available from: http://omega.ilce.edu.mx:3000/ biblioteca/sites/3 milenio/bioet/htm/ sec 5.htm.

6. Goldim JR. Rompendo os limites entre ciência e ética. Episteme, Porto Alegre 2000; (10):5-7.

7. Fortes P, Massarollo MC, Zoboli EL. As experimentações com seres humanos e a Universidade. Jornal da USP 1998; 13(436). 22 a 28 junho. Seção: Opinião. Available from: http://www.usp.br/jorusp/arquivo//998/ jusp436/manchet/rep_res/opinião.html

8. Freitas CBD. Os Comitês de ética em pesquisa: evolução e regulamentação. Bioética 1998; 6(2):189-95. Available from: http:// www. portalmedico.org.br/revista/bio2v6/ cometicapes.htm.
9. Declaração de Helsinque, outubro 2000. Princípios éticos para pesquisa clínica envolvendo seres humanos. Revisada na $52^{\text {a }}$ Assembléia Geral da Associação Médica Mundial. Edimburgo; 2000. Available from: http:// www.pesquisaclínica.bio.br/legislacao/ helsinque.html.

10. Conselho Federal de Medicina. Resolução CFM n. 12/5/85. Available from: http:// www.cfm.org.br.

11. Resolução 023/86. Poder Executivo. Diário Oficial do Estado. São Paulo, SP, n. 177. 1986 set 17. Seção I. p.48.

12. Brasil. Ministério da Saúde. Conselho Nacional de Saúde. Resolução n. 01/88. 13 jun 1988. Dispõe sobre a aprovação das normas de pesquisa em saúde. Diário Oficial da República Federativa do Brasil, Brasília, DF, 126(| |0): 107|3. 1988 jun 14.

13. Pitanguy J. Feminist politics and reproductive rights: the case of Brazil. In: Sen G, Snow RC, editors. Power and decision. The social control of reproduction. Boston: Harvard School of Public Health; 1994. p. I0 I-22.

14. Blaney $\mathrm{CL}$. Long-acting methods require special care. Network 1994; I5(I): 18-21.

15. Blaney CL. Informed consent plays key role. Network 1994; 15(1):22-5

16. Conferência Nacional de Saúde e Direitos da Mulher. Relatório final. Brasília: Centro de Documentação do Ministério da Saúde; 1987. p.57.

17. Brasil. Ministério Nacional da Saúde. Conselho Nacional de Saúde. Resolução 196/96 sobre pesquisa envolvendo seres humanos. Bioética 1996; 4(2): 15-25.

18. Conselho Federal de Medicina. Escolas Médicas: relação de instituições de ensino superior - Curso de Medicina. [citado I 4 jul 2000]. Available from: http://cfm.org.br.

19. PLACIRH. Inventário Latinoamericano de Centros de Investigaciones, Ensenãnza y Recursos Humanos en Reproducción Humana; 1999.

20. Ash DA, Jedrziewski MK, Christakis NA. Response rates to mail surveys published in medical journals. J Clin Epidemiol 1997; 50(I0): I 129-36

21. Kellerman SE, Herold J. Physician response to surveys: a review of the literature. Am J Prev Med 200 I; 20(I):61-7.

Artigo recebido: 08/07/03

Aceito para publicação: 15/I2/2003 